Journal of Mathematics and Statistics 3 (1): 1-11, 2007

ISSN 1549-3644

(C) 2007 Science Publications

\title{
Periodic and Non Periodic (Complex) Behavior of a Model of Bioreactor With Cell Recycling
}

\author{
${ }^{1}$ G. Ibrahim, ${ }^{2}$ M. A. Ramadan, ${ }^{2}$ S. A. A. El-Marouf and ${ }^{2}$ A. M. Al-Mahdi \\ ${ }^{1}$ Basic Engineering Science Department, Faculty of Engineering, Menoufia University, Egypt \\ ${ }^{2}$ Department of Mathematics, Faculty of Science, Menoufia University, Egypt
}

\begin{abstract}
A structured model of bioreactor for an activated sludge process was presented. The stability and bifurcation characteristics of the model are investigated, the bifurcation analysis of the model shows static and complex dynamic behavior (periodic and complex) over a wide range of the model parameters. The model exhibits a new interesting behavior (in some range of parameters) including four static limit points (turning points) and two Hopf points, that cause different kinds and rich of stability characteristics ranging from asymptotically stable and hysteresis to periodic and complex behavior.
\end{abstract}

Key words: Activated sludge process, Structured models, Bifurcation, periodic

\section{INTRODUCTION}

The heterogeneity and the complexity of activated sludge processes pose a continuous challenge to developing models that can incorporate all the necessary levels of information concerning the process and be accurate enough for the adequate control and safe operation of the bioreactor ${ }^{[1]}$.

The complete quantification of the microbiological system on the other requires the understanding of the complex biological and physicochemical interactions in the process and the measurement of a large number of reaction rates, which is often beyond the scope of reasonable measurement techniques. This task is particularly complicated when dynamic modeling is sought. While simple and often hand, unstructured, steady-state models are sufficient for the purpose of plant design, these models are generally inadequate for dynamic simulations and control of the process, since they often fail to predict accurately the effects of process disturbances ${ }^{[2,3]}$. Structured models on the other hand, with different degrees of complexity can supplement the inadequacies of unstructured models. Structured models take into account the inevitable changes in the cell population composition, since the model microbial kinetics are constructed on the basis of at least some of the knowledge accumulated in the vast repository of fundamental biochemistry and microbiology. Structured models, however, may sometimes suffer from over-detailed information that cannot be verified, making them inappropriate for practical use. A good structured model should have a reasonable number of parameters to provide it with some levels of flexibility ${ }^{[4,5]}$.

Besides predicting the effects of external disturbances, a good dynamic model should also be able to predict the different dynamic behaviors the autonomous process may exhibit. Activated sludge reactors have long been known to exhibit a variety of dynamic behavior depending on the process operating conditions ${ }^{[6]}$ for instance, examined both theoretically and experimentally the occurrence of steady-state multiplicity and hysteresis in activated sludge reactors, confirming the experimental findings reported in earlier works ${ }^{[7,8]}$. Bertucoo et al. ${ }^{[9]}$ on the other hand, examined the stability and bifurcation characteristics of the activated sludge reactors with solids recycle and showed the existence of steady-state multiplicity for some range of operating parameters. It is known that over $90 \%$ of municipal wastewater treatment plants rely on activated sludge systems for the core part of the treatment process. These systems encompass biodegradation and sedimentation processes which take place in the aeration and sedimentation tanks respectively the key feature of the biological degradimentation process is that microorganisms i.e., the so-called activated sludge, convert the incoming polluting organism while growing ${ }^{[10]}$.

The effects of substrate inhibited kinetics in activated sludge reactors, has been investigated by many authors ${ }^{[11]}$. It was clearly shown that substrate inhibition models are fundamental in predicting the results of wastewater containing toxic compounds.

The stability and the control of steady state multiplicity in the continuous stirred tank bioreactor (CSTBR) were also the subject of many investigations ${ }^{[6]}$ and investigated the bifurcation and stability of $a(C S T B R)$. Providing a general framework for the analysis of stability and bifurcation mechanisms in the CSTBR with cell recycle using the singularity theory has been investigated ${ }^{[12]}$. A model of 3-dimensional differential equations has been investigated for

Corresponding Author: G. Ibrahim, Basic Engineering Science Department, Faculty of Engineering, Menoufia University, Egypt 
periodicity and bifurcation in $\mathrm{in}^{[1,13-15]}$.

In this work, the dynamic characteristics of a flexible model of activated sludge process with solids recycle are studied. The proposed model is a simplified version of a structured model used by Andrews et al. ${ }^{[1]}$. The model is structured upon substrate and intermediate component growth depending processes.

The model kinetics are based on the other hand, on substrate and intermediate product inhibitory effects. The investigation is based upon the principles of bifurcation theory coupled with continuation techniques which gives more deep analysis to the static and dynamic characteristics of the model.

Process Model: The main processes of the model ${ }^{[1]}$. Substrate $(\mathrm{S}) \rightarrow$ Particulate product $(\mathrm{Xs}) \rightarrow$ Biomass (Xa), is structured upon two processes

1. Formation of an intermediate particulate product (Xs) depending on substrate,

2. Active biomass (Xa) synthesis.

The model is a simplified version of the original Andrews model for activated sludge process where the decay rate is being assumed negligible. This assumption is acceptable when operating at low cell residence time.

The substrate $\mathrm{S}$ is converted to a slowly biodegradable particulate product following the rate expression $U_{1}=\frac{U_{m} S}{S+K_{S}+\alpha S^{2}}$

where $U_{1}$ is the specific rate. This kinetic expression accounts for the well-known phenomenon of substrate inhibition. The Haldane expression has been used for this purpose. The equation requires only three parameters: the specific growth rate $\mathrm{pm}$, the substrate saturation constant $\mathrm{Ks}$ and the substrate inhibition constant $1 / \alpha$.

The Haldane equation is accurate enough to be favored over more complicated inhibitory kinetics models ${ }^{[16]}$. Substrate inhibition has been extensively studied both in batch reactors and in chemostats and it was shown that substrate inhibition models are fundamental in predicting the stability characteristics in activated sludge reactors

(with or without solids recycle), such as the occurrence of steady-state multiplicity and the hysteresis phenomenon $^{[17,18]}$.

Besides the direct inhibitory effects due to the substrate, a delayed inhibitory effect caused by the intermediate product is also assumed. This inhibition effect is assumed to be noncompetitive, i.e., the growth of the intermediate product affects negatively the maximum growth rate $U_{m}$, given by

$U_{m}=\frac{C u 1 K_{i}}{X s+K_{i}}$

where $K i$ is the inhibition constant.

The biomass growth rate on the other hand, depends on the intermediate product following the common Monod behavior:

$U_{2}=\frac{U_{m} X s}{K_{x}+X s}$

where $U_{2}$ is the specific growth rate and $\mathrm{K}_{x}$ is the half saturation constant for biomass synthesis.

The intermediate product inhibition affects then both the substrate uptake rate and the biomass growth rate through the term $U_{m}$ This is in agreement with the observations made in ${ }^{[19]}$ on the influence of particulate intermediate products on the performances of microbial cultures. Equations (1-3) form then the model kinetics. In the following section, the unsteady state component balance equations around the reactor-settler, are written for the different species

* Substrate S. The substrate is consumed to produce the intermediate particulate product Xs. The unsteady state component balance yields

$$
\begin{aligned}
& \mathrm{QS}_{\mathrm{f}}+\mathrm{QRS}-\mathrm{V} \frac{\mathrm{U}_{1}}{\mathrm{Yx} / \mathrm{s}} \\
& \mathrm{Xa}=\mathrm{QWS}+\mathrm{Q}(1+\mathrm{R}-\mathrm{W}) \mathrm{S}+\mathrm{V} \frac{\mathrm{dS}}{\mathrm{dt}},
\end{aligned}
$$

with $\mathrm{Yx} / \mathrm{s}$ is the yield coefficient assumed constant. Equation (4) is also equivalent to

$\theta \frac{d S}{d t}=S_{f}-S-\theta \frac{U_{1}}{Y x / s} X a$

where $\theta=\frac{V}{Q}$ is the reactor residence time.

* Particulate intermediate product X,. The particulate product $\mathrm{X}$, is consumed to produce the biomass. A component balance yields, then

$$
\begin{aligned}
& \mathrm{QXs}_{\mathrm{f}}+\mathrm{QRXs}_{\mathrm{R}}+\mathrm{V}\left(\mathrm{U}_{1}-\mathrm{U}_{2}\right) \mathrm{Xa} \\
& =\mathrm{QWXs}+\mathrm{Q}(1+\mathrm{R}-\mathrm{W}) \mathrm{Xs}+\mathrm{V} \frac{\mathrm{dXs}}{\mathrm{dt}}
\end{aligned}
$$

The assumed ideal conditions in the settler allows the following simple relation between the recycle $\mathrm{Xs}_{R}$ and the effluent $\mathrm{X}$, concentrations

$$
X s_{R}=X s \frac{(1+R-W)}{R}
$$

Then equation (6) becomes

$\theta \frac{d X s}{d t}=X s_{f}-W X s+\theta X a\left(U_{1}-U_{2}\right)$

* Active biomass Xa,. The component balance equation for biomass is

$$
\begin{aligned}
& \mathrm{QXa}_{\mathrm{f}}+\mathrm{QRXa}_{\mathrm{R}}+\mathrm{VU}_{2} \mathrm{Xa} \\
& =\mathrm{QWXa}+\mathrm{Q}(1+\mathrm{R}-\mathrm{W}) \mathrm{Xa}+\mathrm{V} \frac{\mathrm{dXa}}{\mathrm{dt}}
\end{aligned}
$$

Similarly to the intermediate product (equation (7)), a simple relation links the recycle $\mathrm{Xa}_{R}$ and the effluent $\mathrm{Xa}$, biomass concentrations

$$
X a_{R}=X a \frac{(1+R-W)}{R}
$$


which reduces equation (9) to

$$
\theta \frac{d X a}{d t}=X a_{f}-W X a+\theta U_{2} X a
$$

Then autonomous system of the model is

$$
\begin{aligned}
& \theta \frac{d S}{d t}=S_{f}-S-\theta \frac{U_{1}}{Y x / s} X a \\
& \theta \frac{d X s}{d t}=X s_{f}-W X s+\theta X a\left(U_{1}-U_{2}\right) \\
& \theta \frac{d X a}{d t}=X a_{f}-W X a+\theta U_{2} X a
\end{aligned}
$$

Using the formulas of $U_{1}$ and $U_{2}$ as in (1), (2) and (3), system (12) takes the form

$$
\begin{aligned}
& \theta \frac{d S}{d t}=S_{f}-S-\frac{\theta X a}{Y x / s} \frac{\left(C u_{1} K i\right) S}{(X s+K i)\left(K s+S+\alpha S^{2}\right)}, \\
& \theta \frac{\mathrm{dXs}}{\mathrm{dt}}=\mathrm{Xs}_{\mathrm{f}}-\mathrm{WXs}+\theta \mathrm{Xa} \\
& \left\{\frac{\left(\mathrm{Cu}_{1} \mathrm{Ki}\right) \mathrm{S}}{(\mathrm{Xs}+\mathrm{Ki})\left(\mathrm{Ks}+\mathrm{S}+\alpha \mathrm{S}^{2}\right)}-\frac{\left(\mathrm{Cu}_{1} \mathrm{Ki}\right) \mathrm{Xs}}{(\mathrm{Xs}+\mathrm{Ki})(\mathrm{Xs}+\mathrm{Kx})}\right\} \\
& \theta \frac{d X a}{d t}=X a_{f}-W X a+\theta X a \frac{\left(C u_{1} K i\right) X s}{(X s+K i)(X s+K x)},
\end{aligned}
$$

where, $\mathrm{S}, \mathrm{Xs}$ and $\mathrm{Xa}$ are variables, $S_{f}$, $X_{f}, \theta, Y x / s, C u_{1}, K i, K s, K x, \alpha$ and $\mathrm{W}$ are parameters with $X s_{f}=0.8 X_{f}$ and $X a_{f}=0.2 X_{f}$.

The three-dimensional model includes a large number (nine) of parameters. Besides the reactor operating parameters, i.e., feed conditions and purge fraction, the nominal values of the other model parameters are given in Table 1.

Table 1: Nominal values of model parameters

\begin{tabular}{lc}
\hline Parameter & Value \\
\hline $\mathrm{Ki}(\mathrm{mg} / \mathrm{l})$ & 10 \\
$\mathrm{Ks}(\mathrm{mg} / \mathrm{l})$ & 10 \\
$\mathrm{Kx}(\mathrm{mg} / \mathrm{l})$ & 500 \\
$\mathrm{~S}_{f}(\mathrm{mg} / \mathrm{l})$ & 500 \\
$\mathrm{~W}$ & 0.1 \\
$\mathrm{X}_{f}(\mathrm{mg} / \mathrm{l})$ & 100 \\
$\mathrm{Yx} / \mathrm{s} \mathrm{or} \mathrm{Yx}$ & 0.5 \\
$\alpha(1 / \mathrm{mg})$ & 0.11 \\
$\mathrm{Cu} 1(1 / \mathrm{hr})$ & 3 \\
\hline
\end{tabular}

These nominal values (as shown in the table) are taken from realistic ranges given in literature. Among all the model parameters the residence time $\theta$ is the easiest parameter to vary and is chosen as the bifurcation parameter. The bifurcation analysis consists in studying the branching phenomena in the model when its parameters (or a subset of them) are varied around the nominal values.

Numerical tools and presentation techniques: The methodology for static and dynamic bifurcation consists in the numerical continuation techniques, coupled with the principles of bifurcation theory.

The bifurcation diagrams are obtained using the software AUTO of Doedel and Kernevez ${ }^{[20]}$. This package is able to perform both steady state (static) and dynamic bifurcation analysis, including the determination of the entire periodic solution branches. AUTO also computes the Floquet multipliers along periodic solution branches and therefore, determines the stability of the periodic orbits. A periodic orbit loses its stability by a number of mechanisms. The most common of them are period doubling bifurcation and saddle node tangent bifurcation.

Because of periodicity there is always a Floquet multiplier equal to +1 . When Floquet multipliers lie inside the unit circle, the periodic solution is asymptotically stable. A Floquet multiplier leaving the unit circle through -1 indicates period doubling bifurcation. Passage of complex Floquet multipliers out of the unit circle indicates that the periodic orbit bifurcates to an invariant torus.

Periodic solutions can also lose their stability to chaotic attractors. Chaotic attractors show extreme sensitivity to initial conditions and nearby trajectories diverge.

A chaotic attractor is best characterized by its Lyapunov exponents, it has at least one positive Lyapunov exponent. The technique and the algorithm of Wolf et al. ${ }^{[21]}$ are used to efficiently compute these exponents. The DGEAR subroutine ${ }^{[22]}$ with automatic step-size to ensure accuracy for stiff differential equations is used for numerical simulation of periodic as well as chaotic attractors.

\section{RESULTS AND DISCUSSION}

The continuity diagram (substrate concentration $\mathrm{S}$ vs. residence time $\theta$ ) is shown in Fig. 1a for the parameters values shown in Table 1. The continuation diagram in Fig. 1b shows four stable part branches (solid lines) connected to three unstable part branches (dashed lines).

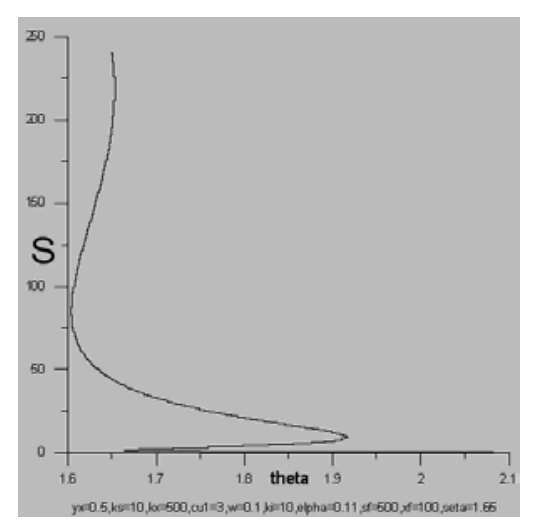

Fig. 1a: Continuity diagram (substrate concentration $\mathrm{S}$ vs. residence time $\theta$ ) 


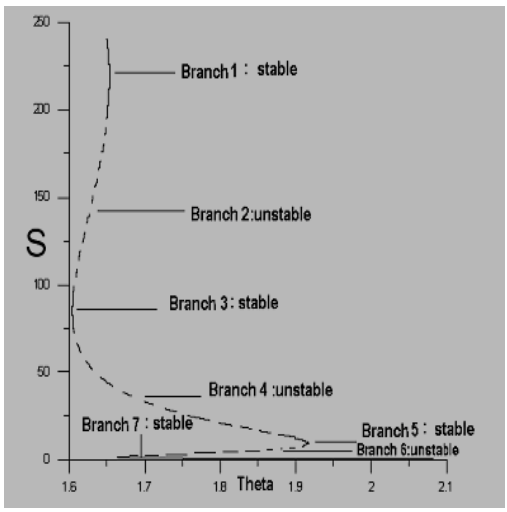

Fig. 1b: Continuity diagram (substrate concentration $\mathrm{S}$ vs. residence time $\theta$ )

Figure 2 shows the existence and the loci of four static limit points SLP (turning points) and two Hopf points (HB), the continuity diagram also shows the periodic solutions birth at $\mathrm{HB}$ points as in Fig. 3, as follows

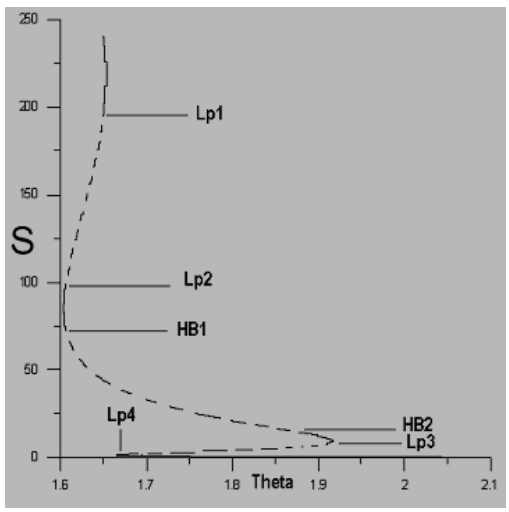

Fig. 2: Continuity diagram (S, $\theta)$

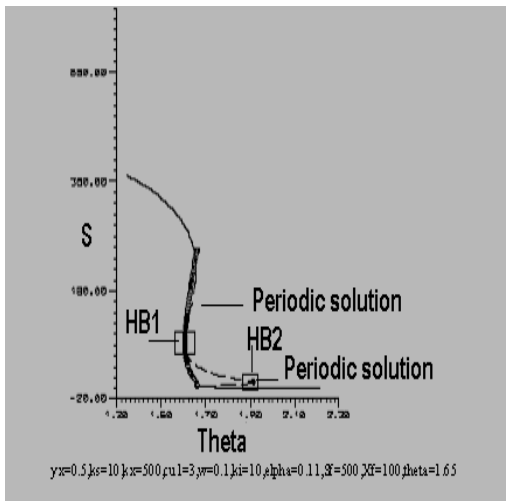

Fig. 3: Periodic solutions appear at HB points

Return to the continuity diagram represent periodic and non periodic branches above we determine four important parts (sub branches) around the limit points and the Hopf points as shown in Fig. 4-7.

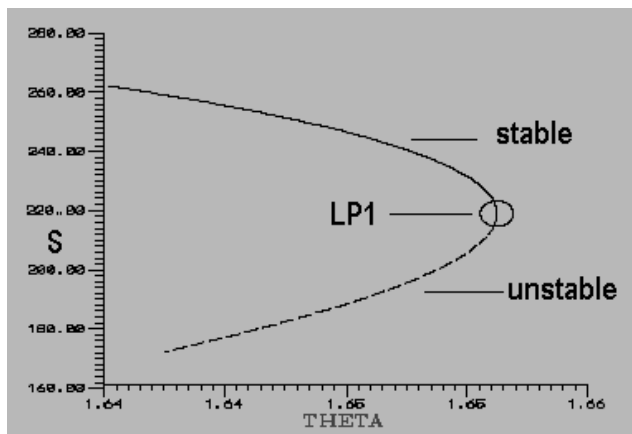

Fig. 4: The vicinity of LP1

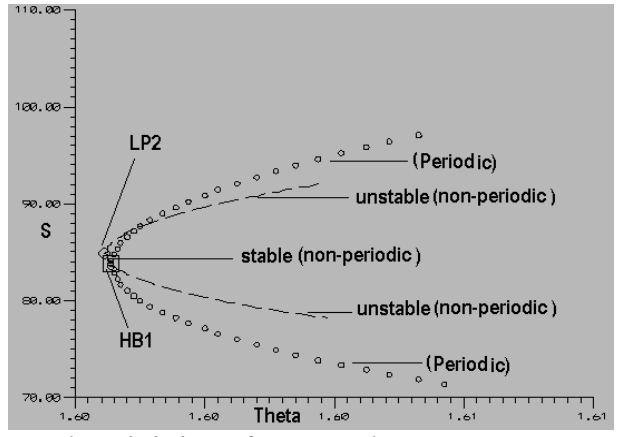

Fig. 5: The vicinity of LP2 and HB1

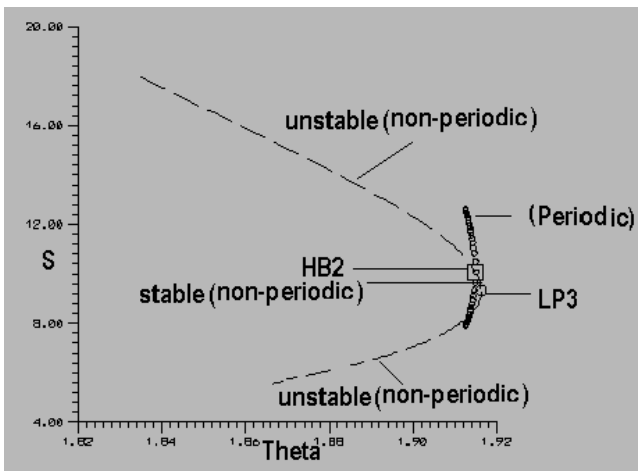

Fig. 6: The vicinity of HB2 and LP3

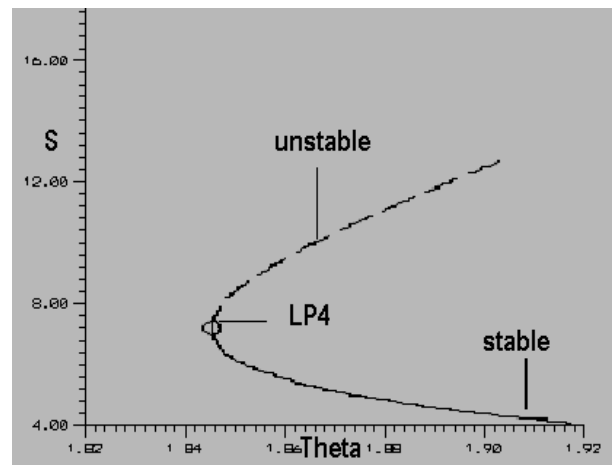

Fig. 7: The vicinity of HB2 and LP3

The existence of Hopf points indicates necessarily the occurrence of an oscillatory behavior in the system. A Hopf point arise when a pair of complex eigenvalues of the Jacobean of the model crosses the imaginary axis transversally. The existence of static limit points and 
Hopf points in the continuation diagram indicates a potential richness in the stability characteristics of the model. It is known from bifurcation theory ${ }^{[23,24]}$ that interactions between a SLP and HB point may lead to a variety of behavior ranging from simple periodicity to complex periodic behavior.

In this analysis, it is helpful to build an overall picture of the possible bifurcation mechanisms that the model may exhibit. This task is best achieved by showing both the loci of the static limit points and the Hopf points in a two-parameter continuation diagram. There are nine figures (two-parameter continuation diagrams) that show the SLP curves and the HB curves as follows

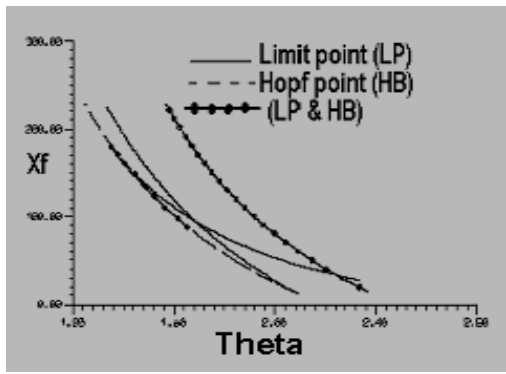

Fig. 8: $(\theta, \mathrm{Xf})$

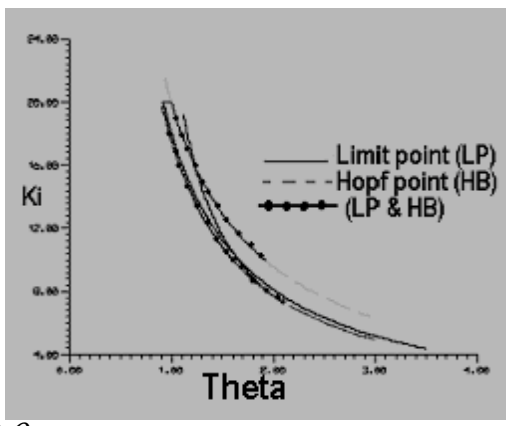

Fig. 9: $(\theta, \mathrm{Ki})$

The above figures of two-parameter continuation showing the loci of the static limit points SLP and the Hopf points HB, solid line is a static limit point, dashed line is a Hopf point and solid-dashed lines is both static limit point and Hopf point.

In the next figures we sketch the other twoparameter continuation of $\mathrm{Ks}, \mathrm{Kx}, \mathrm{Sf}, \mathrm{W}, \mathrm{Cu} 1, \mathrm{Yx}$ and alfa.

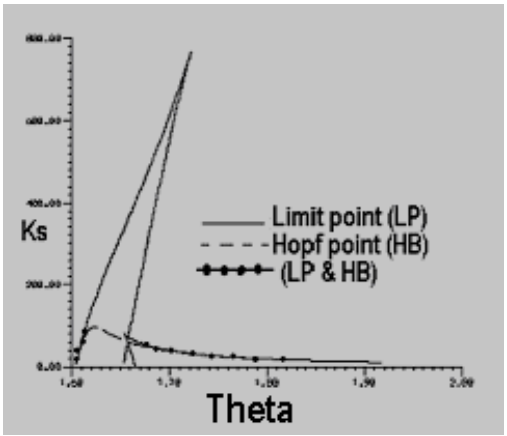

Fig. 10: $(\theta, \mathrm{Ks})$

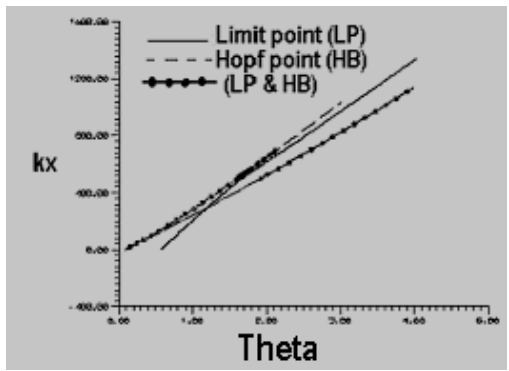

Fig. 11: $(\theta, \mathrm{Kx})$

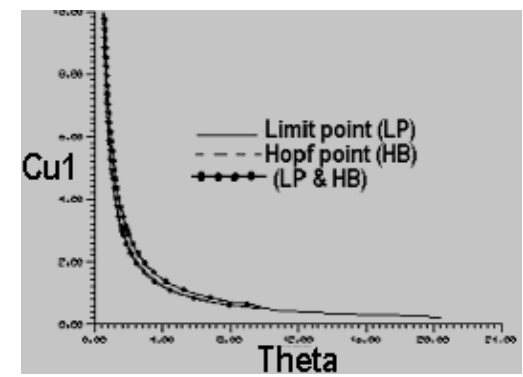

Fig. 12: $(\theta, \mathrm{Cu} 1)$

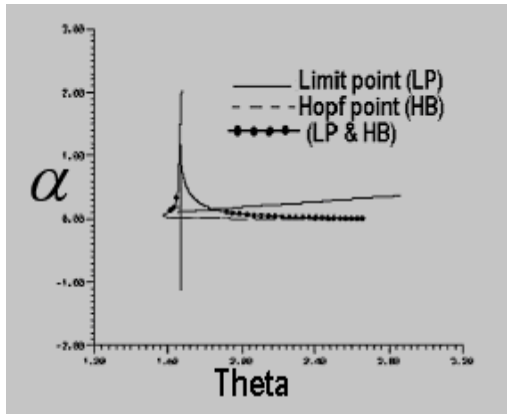

Fig. 13: $(\theta, \alpha)$

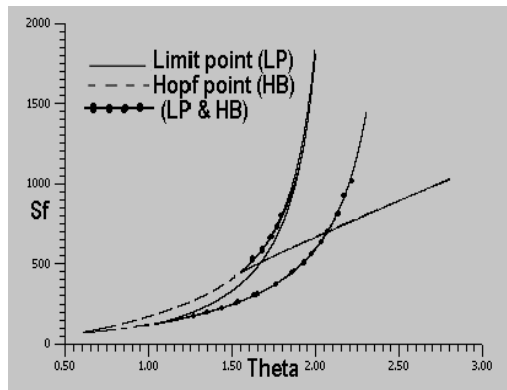

Fig. 14: $(\theta, \mathrm{Sf})$

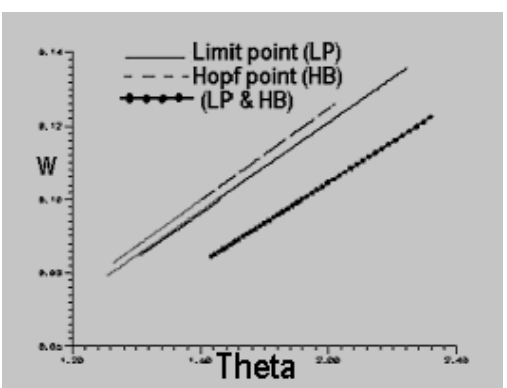

Fig. 15: $(\theta, \mathrm{W})$ 


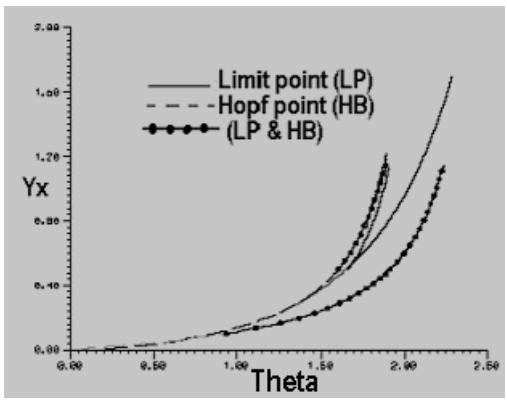

Fig. 16: $(\theta, \mathrm{Yx})$

After that we will investigate one of them in more details i.e. we make partitions in the figure depending on the regions of parameters values that exhibit different behaviors and rest points.

Let we investigate the case of $(\theta, \mathrm{Sf})$ space i.e. Fig. 14 is divided into many regions as shown in Fig. 17 below

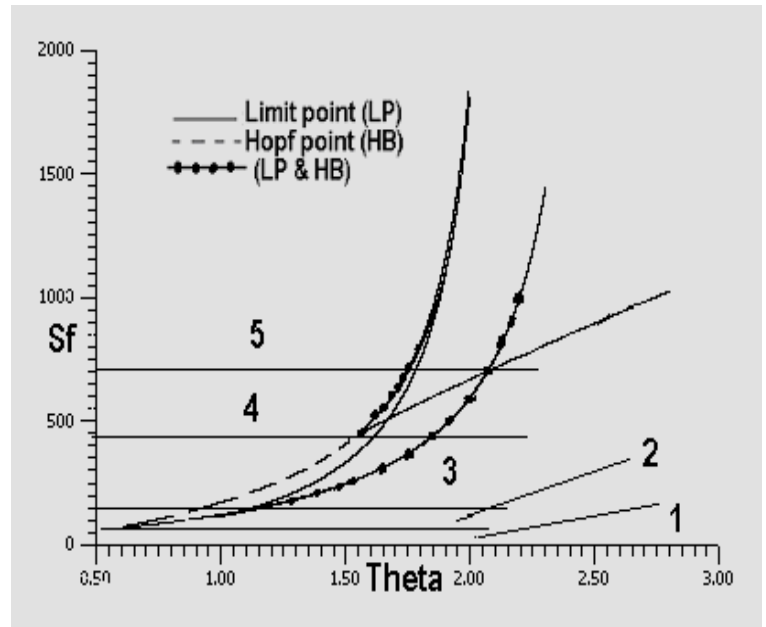

Fig. 17: Partition of two parameter space $(\theta, \mathrm{Sf})$

Figure 17 shows the SLP curves and the HB curves in the parameter space $(\theta, \mathrm{Sf}) .$. It can be seen from this figure that the loci of the $\mathrm{HB}$ points (dashed lines) consist of two lines that form a minimum at point $(\mathrm{Sf}=72.1235)$. An oscillatory behavior should be expected then in the model for any value of the substrate feed concentration larger than the one corresponding to point $(\mathrm{Sf}=72.1235)$.

The loci of the SLP points (solid lines) consist of four lines that form a minimum at point $(\mathrm{Sf}=150)$, At that point two of the SLP lines meet each other, that form a cusp. Static limit points are then expected in the model for any values of feed concentrations higher than the one corresponding to point $(\mathrm{Sf}=150)$. Now before we divide the $(\theta, \mathrm{Sf})$ diagram into many significant parts we give the following notes about that diagram $^{[23,24]}$.

1. The points which is the LP or HB curves form a minimum are special points since they are considered as degenerate points.
2. Interaction between the LP and the HB give a rich characterization in behavior ranging from stable, periodic, non periodic and quasiperiodic to strange attractors and complex.

3. The points of intersections of HB curves with LP curves are also degenerate points, they create the so-called $F_{1}$ degeneracy.

4. The two curves can also be seen to collapse in one line along the right branch of the diagram, this gives birth to an other kind of degeneracy,i.e., the so-called $F_{2}$ degeneracy.

The diagram can be divided into many regions:

Region 1: Simple behavior: This region extended below the value of $\mathrm{Sf}$ that corresponds to the minimum of $\mathrm{HB}$ curves at $\mathrm{Sf}=72.1235$.

This region is characterized by the absence of any limit or Hopf points. A simple behavior(stable solution) is then expected in this region, as showing below.

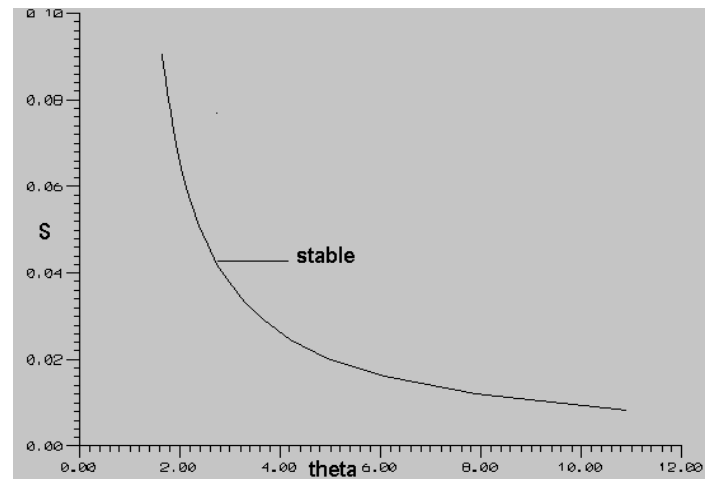

Fig. 18: Continuity diagram $(\theta, \mathrm{S})$

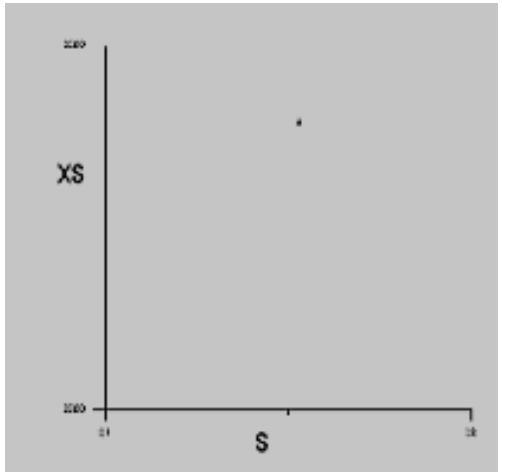

Fig. 19: Phase plane (S,Xs)

Region 2: Periodic behavior: This region extends below the value of $\mathrm{Sf}$ that corresponds to the cusp formed by the $\mathrm{LP}$ curve at $\mathrm{Sf}=150$ and above the point $\mathrm{Sf}=72.1235$ mentioned in region1, which can exhibit periodic solutions, Region 2 is characterized by the appearing of two Hopf points.

Region 3: appearing of hysteresis: This region is setting above region 2 and extends from the cusp formed by the LP' s Curves just below the line $\mathrm{Sf}=410$, 


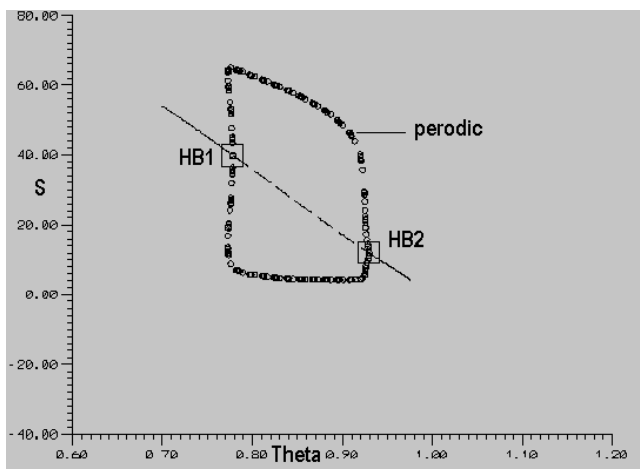

Fig. 20: Continuity diagram, periodic solution $(\mathrm{Sf}=110)$

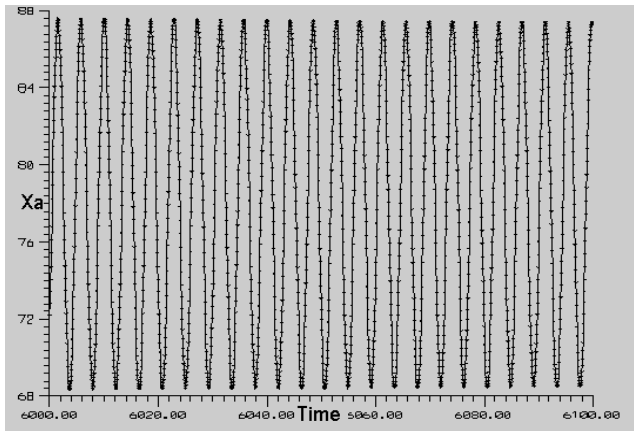

Fig. 21: Time trace (t,Xa), $(\mathrm{Sf}=110)$

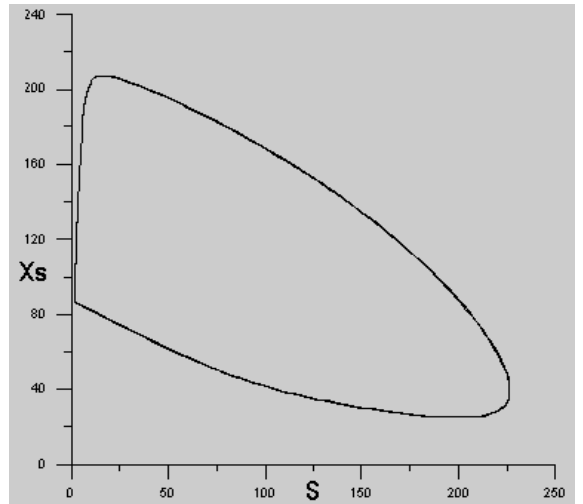

Fig. 22: Phase plane ( $\mathrm{S}, \mathrm{Xs}),(\mathrm{Sf}=110)$

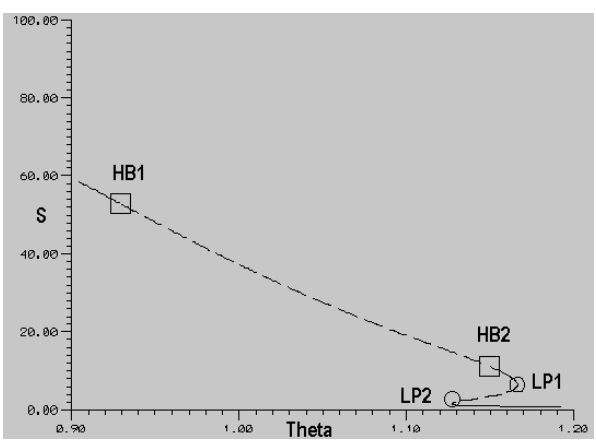

Fig. 23: Continuity diagram: The hysteresis $(\mathrm{Sf}=150)$

which is the beginning of the complex(rich) region that contains many features. Region 3 is characterized by the appearing of two Hopf points and two limit points(turning points).

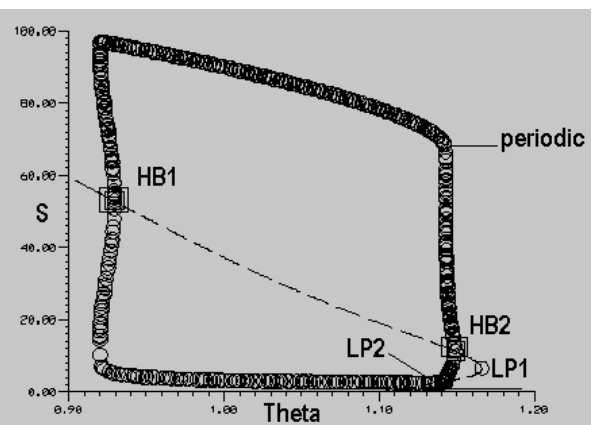

Fig. 24: The hysteresis and periodic solution( $\mathrm{Sf}=150)$

Region 4: Periodic, quasiperiodic and complex: This region is the main in this study since it can be divided itself into many subparts, in this region we have many different characterization of stability, arising from the intersections of LP lines with HB lines(denoted by dashed-dot lines), the presences respectively and the order LP point, LP point, HB point, HB point, LP point and LP point, which is a new result we get in this study than that in ${ }^{[1]}$.

As a complete picture of the continuity diagram, it can be seen as two hysteresis connecting together at any point between $\mathrm{HB} 1$ and HB2, this gives a contraction between that regions.

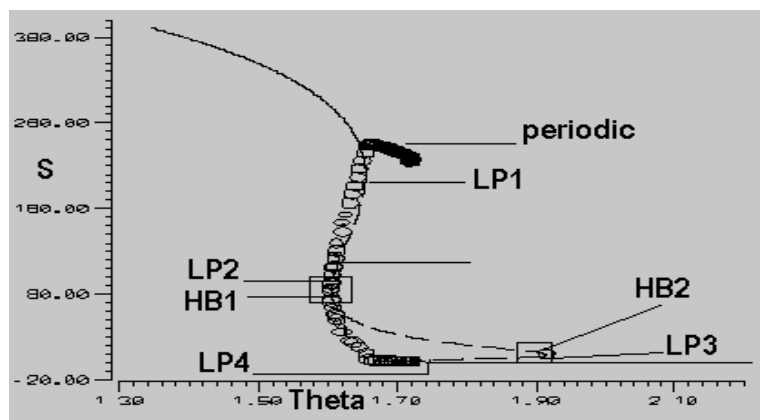

Fig. 25: Continuity diagram, $(\mathrm{Sf}=500)$

The following figures show some behaviors of the model in this region in the order LP point, LP point, HB point, HB point, LP point and LP point, in these figures the behavior is not periodic nor quasi periodic, it is complex

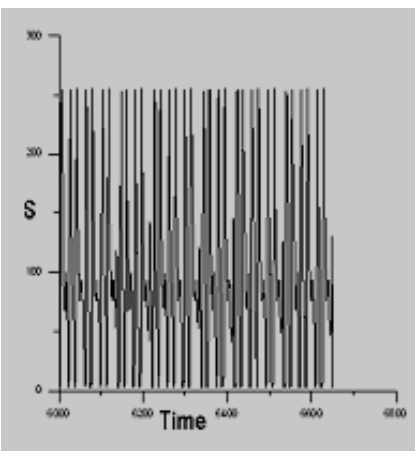

Fig. 26a 


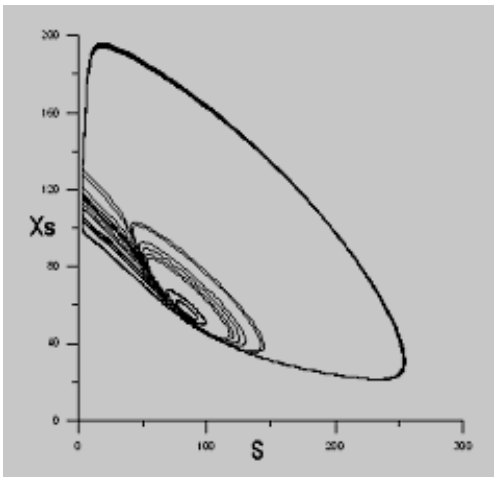

Fig. 26b

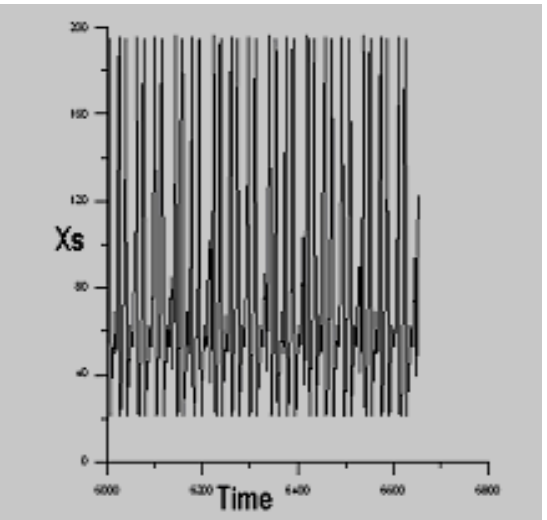

Fig. 26c

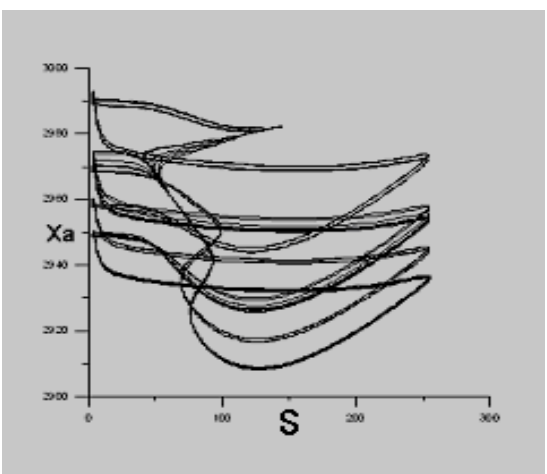

Fig. 26d

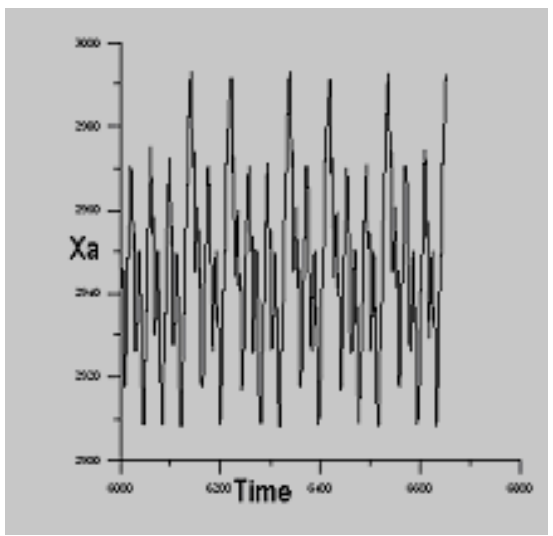

Fig. 26e

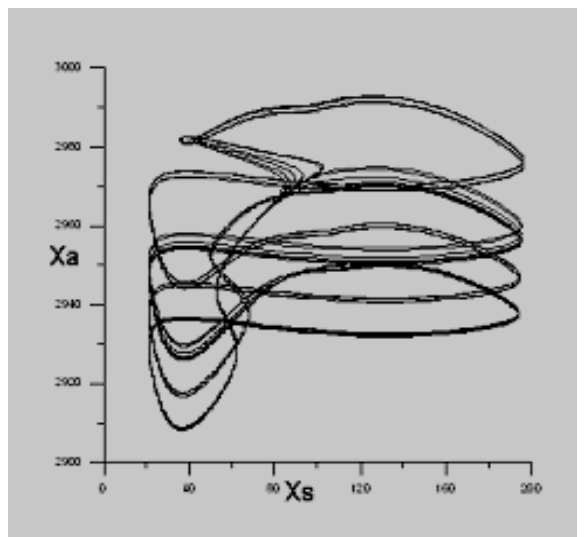

Fig. 26g

$\mathrm{yx}=0.5, \mathrm{ks}=10, \mathrm{kx}=500, \mathrm{cu} 1=3, \mathrm{w}=0.1, \mathrm{ki}=10, \mathrm{alpha}=0.11, \mathrm{Sf}=500, \mathrm{Xf}=10$ 0 , theta $=1.65$

Fig. 26: Time trace and phase plane for non periodic (complex) attractor initiating at LP3 (9.326137, 233.5294, 3219.84)

We can give a clear picture of the behavior shown in Fig. 26e as follows

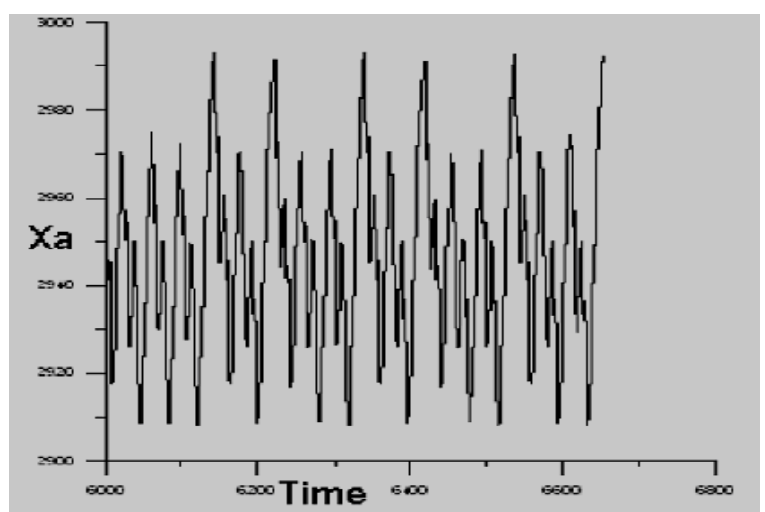

Fig. 27: Enlargement of Fig. 26e

Other picture of this behavior (complex) can be seen with $\mathrm{Sf}=500.45$ and the same values of the other parameters as follows

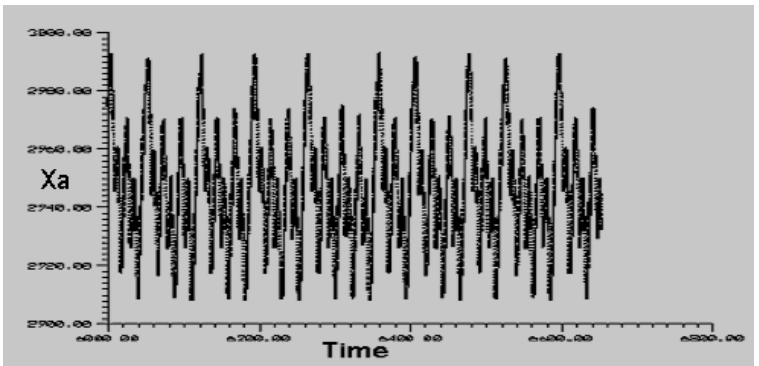

Fig. 28: Time trace for non periodic (complex) behavior

Finally we introduce enlargements for the above diagrams in Fig. 26, first using about 500 iterations as in Fig. 29-32 below 


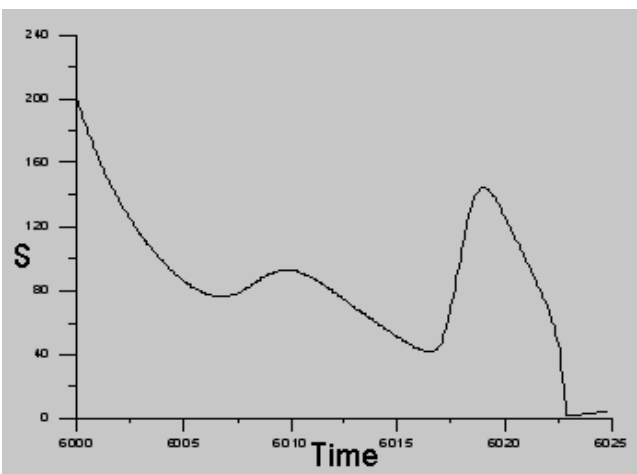

Fig. 29: Enlargement of Fig. 26a

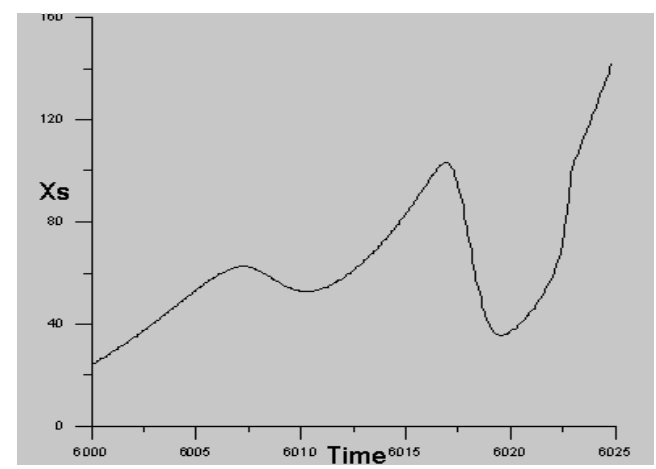

Fig. 30: Enlargement of Fig. 26c

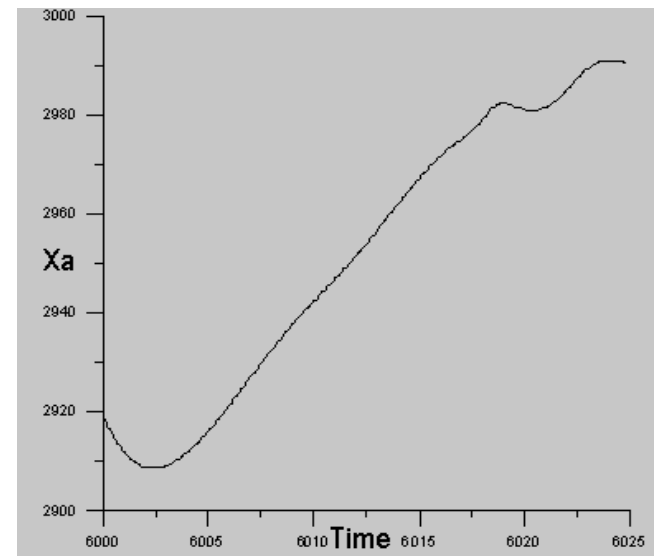

Fig. 31: Enlargement of Fig. 26e

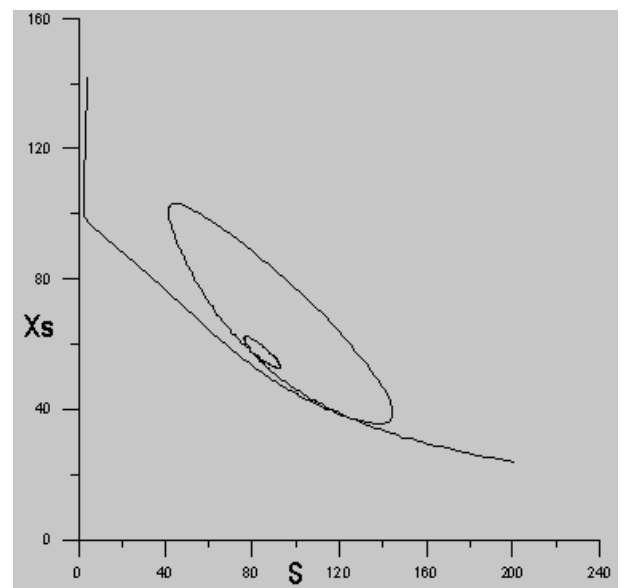

Fig. 32: Enlargement of Fig. 26b
Second, using about 1000 iterations as in Fig. 3336 below

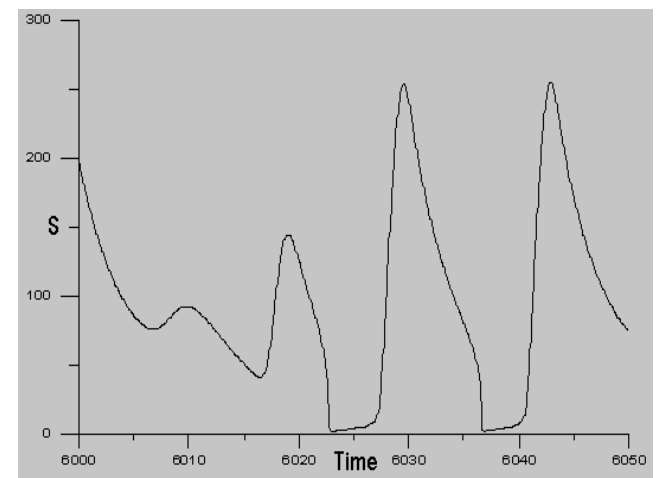

Fig. 33: Enlargement of Fig. 26a

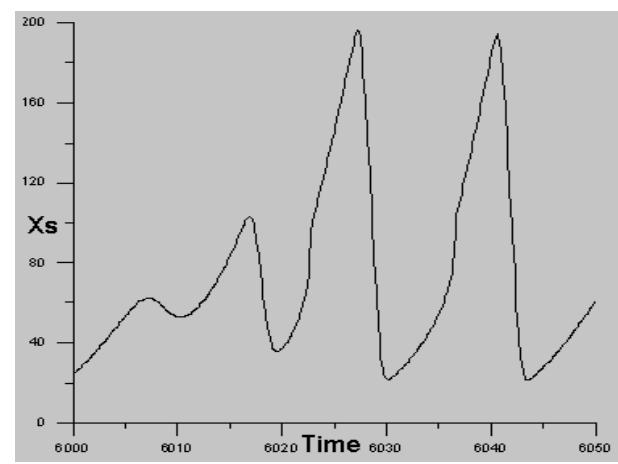

Fig. 34: Enlargement of Fig. 26c

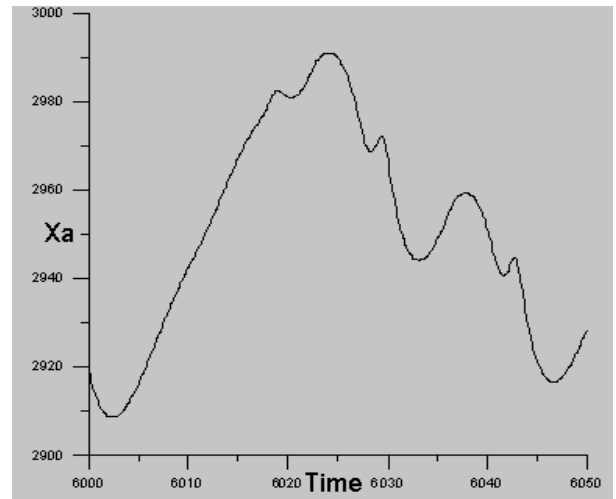

Fig. 35: Enlargement of Fig. 26e

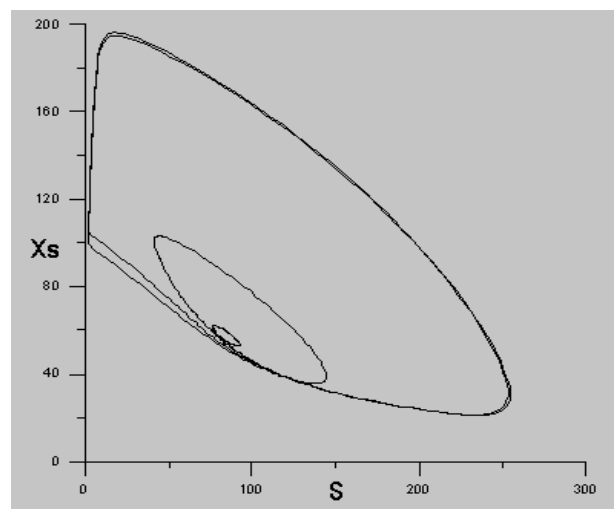

Fig. 36: Enlargement of Fig. 26b 
Third, using about 2000 iterations as in Fig. 37-40 below

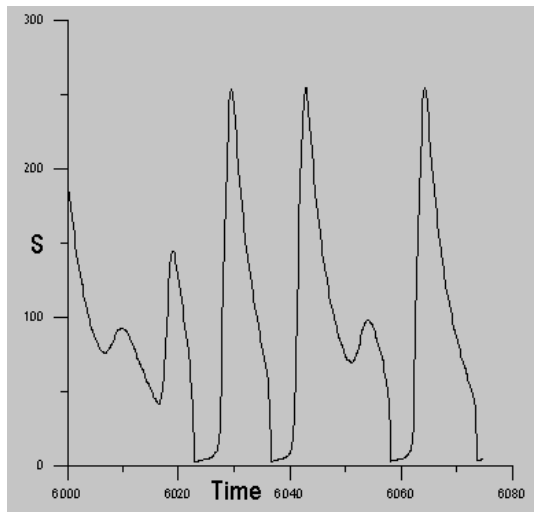

Fig. 37: Enlargement of Fig. 26a

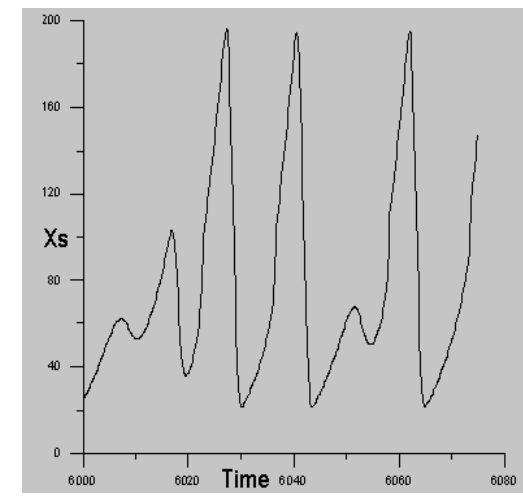

Fig. 38: Enlargement of Fig. 26c

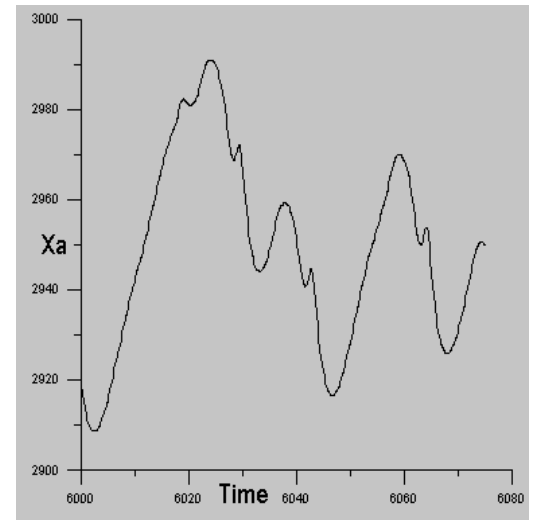

Fig. 39: Enlargement of Fig. 26e

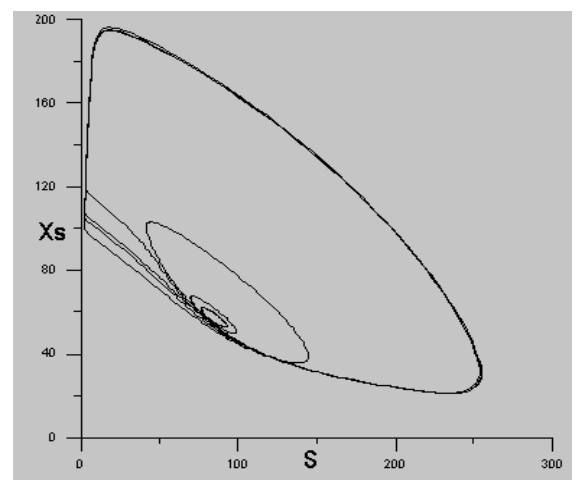

Fig. 40: Enlargement of Fig. 26b
Region 5: Complex behavior: This region is characterized by the appearance of the critical points with the order LP1, HB1, LP2, HB2, LP3, HB2, LP3, LP4 (with $\mathrm{Sf}=800$ ) as shown in Fig. 41.

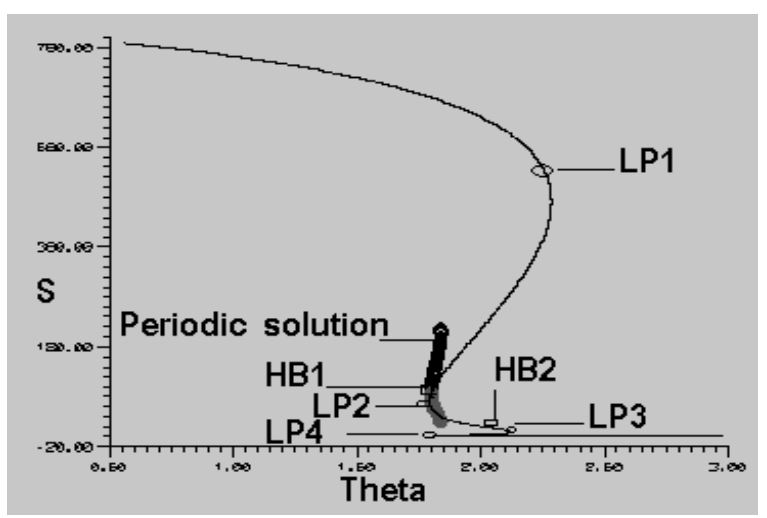

Fig. 41: Continuity diagram, $(\mathrm{Sf}=800)$

\section{CONCLUSION}

In this study, a model for bioreactor of activated sludge process is proposed.

This model is represented by an autonomous system of three non linear first order ordinary differential equations with a set of parameters(ten) and an investigation of the static and dynamic bifurcation of a model was carried out.

This work is a new study for the model with different choosing of the system's parameters, choosing values of parameters as in table 1 gives a new feature of behavior and new stability characteristics, the new region (appearing) is characterized by the presences respectively in the order LP point, LP point, HB point, HB point, LP point and LP point, which is discussed in region 4 .

Other choosing for the parameter values gives different order in appearing of the critical points as in region 5 .

Since the methodology for static and dynamic bifurcation consists of the numerical continuation techniques, coupled with the principles of bifurcation theory, we used a numerical continuation techniques and apply the bifurcation theory to show that the model exhibits a rich stability characteristics ranging from simple Monod-like behavior and hysteresis, to periodic, quasiperiodic and complex behavior.

\section{Nomenclature}

$\mathrm{K} i$ intermediate product inhibition constant (mg/l)

Ks substrate saturation constant (mg/l)

$\mathrm{Kx}$ saturation constant for biomass growth rate $(\mathrm{mg} / \mathrm{l})$

$\mathrm{Q}$ volumetric flow rate $(1 / \mathrm{h})$

$\mathrm{S}$ substrate concentration $(\mathrm{mg} / \mathrm{l})$

$\mathrm{V}$ reactor volume (1)

W sludge withdrawal fraction

Xa biomass concentration (mg/l) 
Xs intermediate particulate product concentration (mg/l)

\section{Greek symbols}

$\alpha$ inverse of substrate inhibition constant $(1 / \mathrm{mg})$

$\theta$ reactor residence time (hr)

$U_{1}$ specific rate for conversion of substrate to intermediate product $\left(\mathrm{hr}^{-1}\right)$

$U_{2}$ specific rate for conversion of intermediate product to active biomass $\left(\mathrm{hr}^{-1}\right)$

$U_{m}$ maximum specific rate $\left(\mathrm{hr}^{-1}\right)$

\section{Subscripts}

$\mathrm{f}$ feed stream

$R$ recycle stream

\section{Abbreviations}

HB Hopf bifurcation point

SLP static limit point

\section{REFERENCES}

1. Ajbar, A. and I. Gamal, 1997. Periodic and non periodic oscillatory behavior in a model for activated sludge reactors. Math. Comput. Model., 25: 9-27.

2. Esener, A.A., T. Veerman, J.A. RoeIs and N.W.F. Kossen, 1982. Biotechnol. Bioeng., 24: 1749.

3. Nielsen, J., K. Nikolajsen and J. Villedsen, 1991. Biotcchnol. Bioeng., 38: 1.

4. Harder, A. and J.A. Roeis, 1982. Adu. B\&hem. Eng. 21: 55.

5. Sheffer, M.S., M. Hiraoka and K. Tsumura, 1984. War. Sci. Tech., 17: 247.

6. DiBiasio, D., H.C. Lim and W.A. Weigand, 1981. An experimental investigation of stability and multiplicity of steady-states in a biological reactor. AIChEJ, 27: 284.

7. Pawlowsky, U. and J.A. Howell, 1973. Bioteehnol. Bioeng., 15: 887.

8. Pawlowsky, U., J.A. Howell and C.T. Chi, 1973. Biotechnol. Bioeng., 15: 905.
9. Bertucco, A., P. Volpe, H.E. Klei, T.F. Anderson and D.W. Sundstrom, 1989. Water. Res., 56: 9.

10. Smets, I.Y., E.N. Banada, J. Deurinck, N. Renders, R. Jenne, J.F. Van Impe, 2006. Dynamic modeling of filamentous bulking in lab-scale activate sludge processes. J. Process Control, 16: 313-319.

11. Agrawal, P., C. Lee, H.C. Lim and D. Ramkrishma, 1982. Theoretical investigations of dynamic behavior of isothermal continuous stirred tank biological reactors. Chem. Engg. Sci., 37: 453-462.

12. Ajbar, A. and I. Gamal, 1997. Stability and bifurcation of an unstructured model of a bioreactor with cell recycle. Math. Comput. Model., 25: 31- 48 .

13. Andrews, J.F., 1974. Wat. Res., 8: 261.

14. Busy, J.B. and J.F. Andrews, 1975. Water Pollut. Control Fed., 47: 1055.

15. Busy, J.B. and J.F. Andrews, 1975. Water Pollut. Control Assoc., 47: 1067.

16. Mulchandani, A. and J.H.T. Luong, 1989. Enzyme Microbial. Technol., 11: 66.

17. Godrej, A.N. and J.H. Sherrard, 19888. Water Pollut. Control Fed., 60: 221.

18. Rozich, A.F. and A.F. Gaudy, 1985. Water Pollut. Control Fed., 57: 795.

19. Rittman, B.E., W. Bae, E. Namkung and C.J. Lu, Wut. Sci. Technol., 19: 517.

20. Doedel, E.J. and J.P. Kerneves, 1986. AUTO: Software for Continuation and Bij+cution Problems in Ordinary Differential Equations. California Institute of Technology, Pasadena, CA.

21. Wolf, A., J.B. Swift, H.L. Swinney and J.A. Vastano, 1985. Physics 16D, 285.

22. Math/PC-Library, IMSL Library, Microsoft, 1992.

23. Guckenheimer, J. and P. Hohns, 1983. Nonlinear Oscillations, Dynamical Systems and Bifurcation of Vector Fields Springer, New York.

24. Wiggins, S., 1990. Introduction to Applied Nonlinear Dynamical Systems and Chaos. Springer-Velag, New York. 